PROCEEDINGS OF THE

AMERICAN MATHEMATICAL SOCIETY

Volume 135, Number 5, May 2007, Pages 1477-1486

S 0002-9939(06)08619-9

Article electronically published on November 14, 2006

\title{
RANDOMIZED UMD BANACH SPACES AND DECOUPLING INEQUALITIES FOR STOCHASTIC INTEGRALS
}

\author{
MARK C. VERAAR \\ (Communicated by N. Tomczak-Jaegermann)
}

\begin{abstract}
In this paper we prove the equivalence of decoupling inequalities for stochastic integrals and one-sided randomized versions of the UMD property of a Banach space as introduced by Garling.
\end{abstract}

\section{INTRODUCTION}

In recent years, decoupling inequalities have been used to construct theories of stochastic integration in UMD Banach spaces [4, 13, 15. The basic idea underlying this approach is to use abstract decoupling inequalities to estimate stochastic integrals

$$
\int_{0}^{T} \phi(t) d W(t),
$$

where $\phi$ is a process with values in a UMD space $E$ and $W$ is a standard Brownian motion, with its decoupled analogue

$$
\int_{0}^{T} \phi(t) d \tilde{W}(t),
$$

where $\tilde{W}$ is a standard Brownian motion independent of $\phi$ and $W$. This decoupled integral is easier to handle, as it is defined in a pathwise sense. Indeed, using a general two-sided decoupling inequality for $E$-valued tangent sequences, McConnell 13. was able to show that a strongly measurable $E$-valued process is stochastically integrable with respect to $W$ if and only if its trajectories, viewed as $E$-valued functions, are stochastically integrable with respect to $\tilde{W}$. His techniques depend heavily on the equivalence of the UMD property and geometric notions related to $\zeta$-convexity. Decoupling inequalities for tangent sequences may be found in [7, 9, 13, 14, 17.

Earlier, Garling [4 had derived a two-sided decoupling inequality for stochastic integrals of elementary $E$-valued processes directly from the definition of the UMD property. More precisely, he proved that a Banach space $E$ is a UMD space if and

Received by the editors September 5, 2005 and, in revised form, December 20, 2005.

2000 Mathematics Subject Classification. Primary 60H05; Secondary 46B09, $46 \mathrm{~B} 20$.

Key words and phrases. Stochastic integration in Banach spaces, randomized UMD spaces, decoupling inequalities, tangent sequences.

The author is supported by the Netherlands Organisation for Scientific Research (NWO) 639.032.201 and by the Research Training Network HPRN-CT-2002-00281.

(C)2006 American Mathematical Society Reverts to public domain 28 years from publication 
only if for some (respectively, for all) $1<p<\infty$ there exist constants $0<c \leq C<$ $\infty$ such that for all elementary $E$-valued processes $\phi$ we have

$$
c \mathbb{E}\left\|\int_{0}^{T} \phi(t) d \tilde{W}(t)\right\|^{p} \leq \mathbb{E}\left\|\int_{0}^{T} \phi(t) d W(t)\right\|^{p} \leq C \mathbb{E}\left\|\int_{0}^{T} \phi(t) d \tilde{W}(t)\right\|^{p} .
$$

These inequalities, combined with the operator-theoretic approach to stochastic integration of Banach space-valued functions developed in [16], was used in [15] to construct a systematic theory of stochastic integration for $E$-valued processes. In particular, necessary and sufficient conditions for $L^{p}$-stochastic integrability were obtained, analogues of the Itô isometry and the Burkholder-Davis-Gundy inequalities were proved, and McConnell's result was recovered as a corollary via standard stopping time arguments.

Various applications of the decoupling inequalities in (1.1) require only one of the two a priori estimates. An analysis of the proof of (1.1) in [4] shows moreover that one-sided decoupling inequalities can be derived from one-sided versions of the UMD property which were introduced subsequently by Garling in [5]. These properties are called $\mathrm{UMD}^{-}$and $\mathrm{UMD}^{+}$below. These properties can be used as in [15] to obtain generalized theories of stochastic integration in which the necessary and sufficient conditions and two-sided estimates for stochastic integrals are replaced by necessary conditions or sufficient conditions, respectively, with one-sided estimates.

The stochastic integration theory in [15] has many consequences and applications. For instance, many results in the theory of stochastic evolution equations in Hilbert spaces (cf. 3 and the references therein), have analogues in $\mathrm{UMD}^{-}$Banach spaces. Therefore, we believe it is important to know the largest class of spaces for which one can construct a stochastic integration theory as in [15. The aim of the present paper is to show that this is the class of $\mathrm{UMD}^{-}$Banach spaces. It is shown that the validity of the second one-sided a priori estimate in (1.1) for all elementary processes implies the $\mathrm{UMD}_{\mathrm{PW}}^{-}$property. With the same ideas one can prove that $E$ has property $\mathrm{UMD}_{\mathrm{PW}}^{+}$if for some $1<p<\infty$ the left estimate in (1.1) holds for all elementary $E$-valued processes, so we include this too. The proofs are based on Skorohod embedding techniques from [4, the Maurey-Pisier characterization of finite cotype and estimates for randomized sums in spaces of finite cotype.

Let $\left(\Omega, \mathcal{F},\left(\mathcal{F}_{n}\right)_{n \geq 1}, \mathbb{P}\right)$ be a filtered probability space, and let $(\tilde{\Omega}, \tilde{\mathcal{F}}, \tilde{\mathbb{P}})$ be a probability space. Both probability spaces are assumed to be rich enough for constructions as below. We shall consider random variables and processes on $(\Omega \times \tilde{\Omega}, \mathcal{F} \times \tilde{\mathcal{F}}, \mathbb{P} \times \tilde{\mathbb{P}})$. On this probability space we use the filtration $\left(\mathcal{F}_{n} \otimes \tilde{\mathcal{F}}\right)_{n \geq 1}$. In most cases our random variables and processes are extensions to $\Omega \times \tilde{\Omega}$ of variables and processes on $\Omega$ or $\tilde{\Omega}$. Integration over $\Omega$ and $\tilde{\Omega}$ will be denoted by $\mathbb{E}$ and $\tilde{\mathbb{E}}$.

Let $\left(r_{n}\right)_{n \geq 1}$ be a Rademacher sequence on $(\Omega, \mathcal{F}, \mathbb{P})$ and let $\mathcal{G}_{0}=\{\emptyset, \Omega\}$ and $\mathcal{G}_{n}=\sigma\left(r_{k}, k=1, \ldots, n\right)$. Recall that a martingale difference sequence $\left(d_{n}\right)_{n=1}^{N}$ is a Paley-Walsh martingale difference sequence if it is a martingale difference sequence with respect to the filtration $\left(\mathcal{G}_{n}\right)_{n=0}^{N}$.

Recall that a Banach space $E$ is a $U M D(p)$ space for $p \in(1, \infty)$ if there exists a constant $C_{p}>0$ such that for every $N \geq 1$, every martingale difference sequence $\left(d_{n}\right)_{n=1}^{N}$ in $L^{p}(\Omega, E)$ and every $\{-1,1\}$-valued sequence $\left(\varepsilon_{n}\right)_{n=1}^{N}$, we have

$$
\left(\mathbb{E}\left\|\sum_{n=1}^{N} \varepsilon_{n} d_{n}\right\|^{p}\right)^{\frac{1}{p}} \leq C_{p}\left(\mathbb{E}\left\|\sum_{n=1}^{N} d_{n}\right\|^{p}\right)^{\frac{1}{p}} .
$$


Similarly, we say $E$ is a $\operatorname{UMD}_{\mathrm{PW}}(p)$-space if one only considers Paley-Walsh martingales in the definition of $\operatorname{UMD}(p)$. In [11, Maurey has shown that $\operatorname{UMD}_{\mathrm{PW}}(p)$ already implies $\operatorname{UMD}(p)$. It was shown by Burkholder in 1 that if $E$ is $\operatorname{UMD}(p)$ space for some $p \in(1, \infty)$, then $E$ is a $\operatorname{UMD}(p)$ space for all $p \in(1, \infty)$. Spaces with this property will be referred to as UMD spaces. For the theory of UMD spaces we refer the reader to 1, 2, and references given therein.

Let $\left(\tilde{r}_{n}\right)_{n \geq 1}$ be a Rademacher sequence on $\tilde{\Omega}$.

Definition 1.1. Let $E$ be a Banach space and let $p \in(1, \infty)$.

(1) The space $E$ is a $U M D_{\mathrm{PW}}^{-}(p)$ space if there exists a constant $C_{p}^{-}>0$ such that for every $N \geq 1$ and every Paley-Walsh martingale difference sequence $\left(d_{n}\right)_{n=1}^{N}$ in $L^{p}(\Omega, E)$, we have

$$
\left(\mathbb{E}\left\|\sum_{n=1}^{N} d_{n}\right\|^{p}\right)^{\frac{1}{p}} \leq C_{p}^{-}\left(\mathbb{E} \tilde{\mathbb{E}}\left\|\sum_{n=1}^{N} \tilde{r}_{n} d_{n}\right\|^{p}\right)^{\frac{1}{p}} .
$$

(2) The space $E$ is a $U M D_{\mathrm{PW}}^{+}(p)$ space if there exists a constant $C_{p}^{+}>0$ such that for every $N \geq 1$ and every Paley-Walsh martingale difference sequence $\left(d_{n}\right)_{n=1}^{N}$ in $L^{p}(\Omega, E)$, we have

$$
\left(\mathbb{E} \tilde{\mathbb{E}}\left\|\sum_{n=1}^{N} \tilde{r}_{n} d_{n}\right\|^{p}\right)^{\frac{1}{p}} \leq C_{p}^{+}\left(\mathbb{E}\left\|\sum_{n=1}^{N} d_{n}\right\|^{p}\right)^{\frac{1}{p}} .
$$

The corresponding notion of $\mathrm{UMD}^{-}$and $\mathrm{UMD}^{+}$spaces, where arbitrary martingale difference sequences are allowed, has been studied by Garling in [5]. It was shown there that if $E$ is a $\operatorname{UMD}^{ \pm}(p)$ space for some $p \in(1, \infty)$, then $E$ is a $\operatorname{UMD}^{ \pm}(p)$ space for all $p \in(1, \infty)$. Thus, both definitions are independent of $p \in(1, \infty)$ and spaces with this property will be referred to as $U M D^{-}$and $U M D^{+}$ spaces. In [5] these properties are called LERMT (Lower Estimates for Random Martingale Transforms) and UERMT (Upper Estimates for Random Martingale Transforms) respectively. We preferred the notation $\mathrm{UMD}^{-}$and $\mathrm{UMD}^{+}$, since it emphasizes the relation with UMD. Here the superscript ${ }^{-}$stands for Lower and the superscript ${ }^{+}$stands for Upper. Similarly, one can show that $\operatorname{UMD}_{\mathrm{PW}}^{ \pm}(p)$ are $p$-independent and these will denoted by $U M D_{\mathrm{PW}}^{ \pm}$. It seems to be an open problem if $\mathrm{UMD}_{\mathrm{PW}}^{-}$implies $\mathrm{UMD}^{-}$and if $\mathrm{UMD}_{\mathrm{PW}}^{+}$implies $\mathrm{UMD}^{+}$.

We list some results on $\mathrm{UMD}^{-}$and $\mathrm{UMD}^{+}$spaces, the proofs of which can be found in 5]:

- If $E$ is a $\mathrm{UMD}^{+}$space, then its dual $E^{*}$ is a $\mathrm{UMD}^{-}$space. If $E^{*}$ is a $\mathrm{UMD}^{-}$ space, then its predual $E$ is a $\mathrm{UMD}^{+}$space.

- Every $\mathrm{UMD}^{-}$space has finite cotype. Every $\mathrm{UMD}^{+}$space is super-reflexive.

- $E$ is a UMD space if and only if it is both $\mathrm{UMD}^{-}$and $\mathrm{UMD}^{+}$.

Similar results hold for $\mathrm{UMD}_{\mathrm{PW}}^{-}$and $\mathrm{UMD}_{\mathrm{PW}}^{+}$spaces.

It was shown in [5] that $l^{1}$ is a $\mathrm{UMD}^{-}$space. It can be shown that if $E$ is a $\mathrm{UMD}^{-}$space and if $(S, \Sigma, \mu)$ is a $\sigma$-finite measure space, then $L^{p}(\Omega ; E)$ is a $\mathrm{UMD}^{-}$ space for all $p \in[1, \infty)$. A similar result holds for $\mathrm{UMD}^{+}$for $p \in(1, \infty)$.

Apart from trivial cases, the space $L^{1}(S, \mu)$ is an example of a $\mathrm{UMD}^{-}$space that is not UMD. It appears to be unknown if there exist $\mathrm{UMD}^{+}$or $\mathrm{UMD}_{\mathrm{PW}}^{+}$spaces that are not UMD (cf. [6, Problem 4.2]). 


\section{MAIN RESUlt}

Let $W$ be a Brownian motion on $(\Omega, \mathcal{F}, \mathbb{P})$ and let $\left(\mathcal{F}_{t}\right)_{t \geq 0}$ be the augmented filtration induced by $W$. Similarly, let $\tilde{W}$ be a Brownian motion on $(\tilde{\Omega}, \tilde{\mathcal{F}}, \tilde{\mathbb{P}})$ and let $\left(\tilde{\mathcal{F}}_{t}\right)_{t \geq 0}$ be the augmented filtration induced by $\tilde{W}$.

Let $E$ be a real Banach space. A process $\phi:[0, \infty) \times \Omega \rightarrow E$ will be called an elementary process if it is of the form

$$
\phi(t, \omega)=\mathbf{1}_{[0]}(t) \xi_{0}(\omega)+\sum_{n=1}^{N} \mathbf{1}_{\left(t_{n-1}, t_{n}\right]}(t) \xi_{n}(\omega),
$$

where $0 \leq t_{0}<\cdots<t_{N}<\infty, \xi_{n}$ is an elementary $\mathcal{F}_{t_{n-1}}$-measurable random variable, $n=1, \ldots, N$ and $\xi_{0}$ is $\mathcal{F}_{0}$-measurable. The stochastic integral $\int_{0}^{\infty} \phi(t) d W(t)$ is defined in the usual way and is an element of $L^{p}(\Omega ; E)$ for all $p \in[1, \infty)$.

Theorem 2.1 (Garling). For a UMD space $E$ and $p \in(1, \infty)$ the following statements hold:

(1) There exists a constant $c_{p}>0$ such that for all elementary processes $\phi$,

$$
\mathbb{E}\left\|\int_{0}^{\infty} \phi(t) d W(t)\right\|^{p} \leq c_{p}^{p} \mathbb{E} \tilde{\mathbb{E}}\left\|\int_{0}^{\infty} \phi(t) d \tilde{W}(t)\right\|^{p} .
$$

(2) There exists a constant $c_{p}>0$ such that for all elementary processes $\phi$,

$$
\mathbb{E} \tilde{\mathbb{E}}\left\|\int_{0}^{\infty} \phi(t) d \tilde{W}(t)\right\|^{p} \leq c_{p}^{p} \mathbb{E}\left\|\int_{0}^{\infty} \phi(t) d W(t)\right\|^{p} .
$$

Conversely, if (2.1) and (2.2) hold for all elementary processes $\phi$, then $E$ is a UMD space.

Inspection of the proof in [4, Theorem 2] shows that (2.1) only requires $\mathrm{UMD}^{-}$ and (2.2) only requires $\mathrm{UMD}^{+}$. The main result of this paper reads as follows.

Theorem 2.2. Let $E$ be a Banach space $E$ and let $p \in(1, \infty)$. The following statements hold:

(1) If there exists a constant $c_{p}>0$ such that (2.1) holds for all elementary processes, then $E$ is a $U M D_{\mathrm{PW}}^{-}$space.

(2) If there exists a constant $c_{p}>0$ such that (2.2) holds for all elementary processes, then $E$ is a $U M D_{\mathrm{PW}}^{+}$space.

Although these results are in some sense not surprising, they appear to be new and nontrivial to prove.

For the proof we need some lemmas. The first lemma is well known and follows from the strong Markov property.

Lemma 2.3. Let $\tau_{0}=0$ and define inductively

$$
\tau_{n}=\inf \left\{t \geq \tau_{n-1}:\left|W_{t}-W_{\tau_{n-1}}\right|=1\right\}, \quad 1 \leq n \leq N .
$$

Then $\left(\tau_{n}\right)_{n=1}^{N}$ is an increasing sequence of stopping times and $\left(\Delta \tau_{n}, \Delta W_{n}\right)_{n=1}^{N}$ is an i.i.d. sequence of random vectors, where

$$
\Delta \tau_{n}=\tau_{n}-\tau_{n-1}, \quad \Delta W_{n}=W_{\tau_{n}}-W_{\tau_{n-1}}, \quad 1 \leq n \leq N .
$$

Moreover $\left(\Delta W_{n}\right)_{n=1}^{N}$ is a Rademacher sequence adapted to $\left(\mathcal{F}_{\tau_{n}}\right)_{n=1}^{N}$. 
The next lemma gives some important properties of the independent Brownian motion $\tilde{W}$ at random times. Such stopped Brownian motions $\tilde{W}$ are not Gaussian random variables in general, but in this case they inherit some important properties.

Lemma 2.4. For $1 \leq n \leq N$, let $\Delta \tilde{W}_{n}=\tilde{W}_{\tau_{n}}-\tilde{W}_{\tau_{n-1}}$. Then $\left(\Delta \tilde{W}_{n}\right)_{n=1}^{N}$ is an i.i.d. sequence of symmetric random variables, which is independent of $\left(\Delta W_{n}\right)_{n=1}^{N}$. Furthermore, each $\Delta W_{n}$ has finite moments of all orders.

Proof. For all $1 \leq n \leq N, \Delta \tilde{W}_{n}$ is symmetric, because $\Delta \tilde{W}_{n}(\omega, \cdot)$ is symmetric for each $\omega \in \Omega$. It follows from the strong Markov property of $(W, \tilde{W})$ that $\left(\Delta W_{n}, \Delta \tilde{W}_{n}\right)_{n=1}^{N}$ is an i.i.d. sequence. So in order to prove the independence of $\left(\Delta \tilde{W}_{n}\right)_{n=1}^{N}$ and $\left(\Delta W_{n}\right)_{n=1}^{N}$, it is enough to show that $\Delta W_{1}=W_{\tau_{1}}$ and $\Delta \tilde{W}_{1}=\tilde{W}_{\tau_{1}}$ are independent. The following argument is shown to us by Tuomas Hytönen. For every Brownian motion $B$ on $\Omega$ we introduce the following two stopping times:

$$
\tau_{ \pm}^{B}=\inf \left\{t \geq 0: B_{t}= \pm 1\right\}
$$

Note that $\tau_{1}=\tau_{-}^{W} \wedge \tau_{+}^{W}$ and for the Brownian motion $-W$, we have $\tau_{+}^{-W}=\tau_{-}^{W}$ and $\tau_{-}^{-W}=\tau_{+}^{W}$. Let $B \in \mathbb{R}$ be some Borel measurable set. Since $(W, \tilde{W})$ is identically distributed with $(-W, \tilde{W})$ it follows that

$$
\begin{aligned}
& \mathbb{P}\left(W_{\tau_{1}}=1, \tilde{W}_{\tau_{1}} \in B\right)=\mathbb{P}\left(\tau_{+}^{W}<\tau_{-}^{W}, \tilde{W}_{\tau_{1}} \in B\right) \\
& \quad=\mathbb{P}\left(\tau_{-}^{-W}<\tau_{+}^{-W}, \tilde{W}_{\tau_{1}} \in B\right)=\mathbb{P}\left(W_{\tau_{1}}=-1, \tilde{W}_{\tau_{1}} \in B\right) .
\end{aligned}
$$

Clearly,

$$
\mathbb{P}\left(W_{\tau_{1}}=1, \tilde{W}_{\tau_{1}} \in B\right)+\mathbb{P}\left(W_{\tau_{1}}=-1, \tilde{W}_{\tau_{1}} \in B\right)=\mathbb{P}\left(\tilde{W}_{\tau_{1}} \in B\right) .
$$

Hence

$$
\mathbb{P}\left(W_{\tau_{1}}=1, \tilde{W}_{\tau_{1}} \in B\right)=\frac{1}{2} \mathbb{P}\left(\tilde{W}_{\tau_{1}} \in B\right)=\mathbb{P}\left(W_{\tau_{1}}=1\right) \mathbb{P}\left(\tilde{W}_{\tau_{1}} \in B\right) .
$$

The same holds for -1 . This proves the independence.

For $0<p<\infty$, we have

$$
\mathbb{E} \tilde{\mathbb{E}}\left|\Delta \tilde{W}_{n}\right|^{p}=\mathbb{E} \tilde{\mathbb{E}}\left|\tilde{W}_{\tau_{1}}\right|^{p}=g_{p}^{p} \mathbb{E} \tau_{1}^{p / 2},
$$

where $g_{p}^{p}$ is the $p$-th moment of a standard Gaussian random variable and the statement follows from the elementary fact that $\tau_{1}$ has finite moments of all orders.

Below we will consider adapted and measurable processes $\phi:[0, \infty) \times \Omega \rightarrow E$ that take values in a finite-dimensional subspace of $E$. Since $n$-dimensional subspaces of $E$ are isomorphic to $\mathbb{R}^{n}$, one may construct the stochastic integral for such processes $\phi$ that satisfy $t \mapsto \phi(t, \omega) \in L^{2}(0, \infty, E)$ for almost all $\omega \in \Omega$. By the Burkholder-Davis-Gundy inequalities we have for all $p \in(1, \infty)$ and for $\phi$ as above, $\int_{0}^{\infty} \phi(t) d W(t) \in L^{p}(\Omega ; E)$ if $\phi \in L^{p}\left(\Omega ; L^{2}(0, \infty ; E)\right)$. In this case the decoupled stochastic integral $\int_{0}^{\infty} \phi(t) d \tilde{W}(t)$ is defined pathwise as an element of $L^{p}\left(\Omega ; L^{p}(\tilde{\Omega} ; E)\right)$. Moreover, if (2.1) or (2.2) holds for all elementary processes one may extend this to all processes as above. In fact, Garling proved (2.1) and (2.2) for this class of processes.

The next lemma is a variation of an example in $[5$. We include a proof for convenience. 
Lemma 2.5. Let $E=c_{0}$ and $p \in[1, \infty)$. There does not exist a constant $c_{p}>0$ such that for all elementary processes $\phi$, (2.1) holds.

Proof. Assume there exists a constant $c_{p}>0$ such that for all elementary processes $\phi$, (2.1) holds. Then we may extend (2.1) to all measurable and adapted processes $\phi \in L^{p}\left(\Omega ; L^{2}(0, \infty ; E)\right)$ that take values in a finite-dimensional subspace of $E$. For each $N \geq 1$, we will construct a process $\phi$ as above and such that

$$
\left(\mathbb{E}\left\|\int_{0}^{\infty} \phi(t) d W(t)\right\|^{p}\right)^{1 / p}=N \text { and }\left(\mathbb{E} \tilde{\mathbb{E}}\left\|\int_{0}^{\infty} \phi(t) d \tilde{W}(t)\right\|^{p}\right)^{1 / p} \leq K_{p} \sqrt{N} .
$$

Here $K_{p}>0$ is some universal constant. This gives a contradiction.

We modify an example in [5] in such a way that the martingale differences arise as stochastic integrals. We use the notation of Lemmas 2.3 and 2.4. Fix an integer $N \geq 1$. Let $D=\{-1,1\}^{N}$, and for each $e=\left(e_{n}\right)_{n=1}^{N} \in D$ define the process $\phi_{e}:[0, \infty) \times \Omega \rightarrow \mathbb{R}$ by

$$
\phi_{e}(t)= \begin{cases}e_{n} \mathbf{1}_{A_{e, n}} & \text { for } t \in\left(\tau_{n-1}, \tau_{n}\right], n=1, \ldots, N, \\ 0 & \text { for } t=0 \text { or } t>\tau_{N},\end{cases}
$$

where $A_{e, 1}=\Omega$ and for $2 \leq n \leq N$,

$$
A_{e, n}=\left\{\Delta W_{1}=e_{1}, \ldots, \Delta W_{n-1}=e_{n-1}\right\} .
$$

Then each $\phi_{e}$ is stochastically integrable with

$$
\int_{0}^{\infty} \phi_{e}(t) d W(t)=\sum_{n=1}^{N} \Delta W_{n} e_{n} \mathbf{1}_{A_{e, n}} .
$$

Define $\phi:[0, \infty) \times \Omega \rightarrow l^{\infty}(D)$ by $\phi=\left(\phi_{e}\right)_{e \in D}$. Then $\phi$ is stochastically integrable and for almost all $\omega \in \Omega$ and $e \in D$ we have $\left|\left(\int_{0}^{\infty} \phi(t) d W(t)\right)(\omega)(e)\right| \leq N$. For almost all $\omega \in \Omega$ and $e=\left(\Delta W_{n}(\omega)\right)_{n=1}^{N}$ we have $\left|\left(\int_{0}^{\infty} \phi(t) d W(t)\right)(\omega)(e)\right|=N$. This shows that

$$
\left(\mathbb{E}\left\|\int_{0}^{\infty} \phi(t) d W(t)\right\|_{l^{\infty}(D)}^{p}\right)^{1 / p}=N, \text { for all } p \in[1, \infty) .
$$

On the other hand, we have

$$
\int_{0}^{\infty} \phi(t) d \tilde{W}(t)=\sum_{n=1}^{N} \Delta \tilde{W}_{n} v_{n}
$$

where for $1 \leq n \leq N, v_{n}=\left(e_{n} \mathbf{1}_{A_{e, n}}\right)_{e \in D}$.

For $\omega \in \Omega$ and $e \in D$ let $k(\omega, e)$ be 0 if $\Delta W_{1}(\omega) \neq e_{1}$ and let $k(\omega, e)$ be the maximum of all integers $n \leq N$ such that $\Delta W_{i}(\omega)=e_{i}$ for all $i \leq n$ if $\Delta W_{1}(\omega)=e_{1}$. For almost all $\omega \in \Omega$ and for all $e \in D,\left(\sum_{n=1}^{N} \Delta \tilde{W}_{n} v_{n}\right)(\omega)(e)$ is equal to

$$
\begin{array}{cc}
-\Delta \tilde{W}_{k(\omega, e)+1}(\omega, \cdot) \Delta W_{k(\omega, e)+1}(\omega)+\sum_{n=1}^{k(\omega, e)} \Delta \tilde{W}_{n}(\omega, \cdot) \Delta W_{n}(\omega), & \text { if } k(\omega, e)<N, \\
\sum_{n=1}^{N} \Delta \tilde{W}_{n}(\omega, \cdot) \Delta W_{n}(\omega), & \text { if } k(\omega, e)=N .
\end{array}
$$

Of course we have for all $k \leq N$,

$$
-\tilde{W}_{k} \Delta W_{k}+\sum_{n=1}^{k-1} \Delta \tilde{W}_{n} \Delta W_{n}=2 \sum_{n=1}^{k-1} \Delta \tilde{W}_{n} \Delta W_{n}-\sum_{n=1}^{k} \Delta \tilde{W}_{n} \Delta W_{n} .
$$


We obtain that for almost all $\omega \in \Omega$,

$$
\left\|\int_{0}^{\infty} \phi(t, \omega) d \tilde{W}(t)\right\|_{l^{\infty}(D)} \leq 3 \sup _{k \leq N}\left|\sum_{n=1}^{k} \Delta \tilde{W}_{n}(\omega, \cdot) \Delta W_{n}(\omega)\right| .
$$

Since for almost all $\omega \in \Omega,\left(\Delta \tilde{W}_{n}(\omega, \cdot)\right)_{n=1}^{N}$ is a sequence of independent centered Gaussian random variables on $\tilde{\Omega}$, we have by the Lévy-Octaviani inequalities for independent symmetric random variables (see [9, Section 1.1]) for almost all $\omega \in \Omega$,

$$
\begin{gathered}
\tilde{\mathbb{E}} \sup _{k \leq N}\left|\sum_{n=1}^{k} \Delta \tilde{W}_{n}(\omega, \cdot) \Delta W_{n}(\omega)\right|^{p} \leq 2^{p} \tilde{\mathbb{E}}\left|\sum_{n=1}^{N} \Delta \tilde{W}_{n}(\omega, \cdot) \Delta W_{n}(\omega)\right|^{p} \\
=2^{p} \tilde{\mathbb{E}}\left|\sum_{n=1}^{N} \Delta \tilde{W}_{n}(\omega, \cdot)\right|^{p}=2^{p} \tilde{\mathbb{E}}\left|\tilde{W}_{\tau_{N}}(\omega, \cdot)\right|^{p}=2^{p} g_{p}^{p} \tau_{N}(\omega)^{p / 2}
\end{gathered}
$$

Here $g_{p}^{p}$ is the $p$-th moment of a standard Gaussian random variable. We may conclude that

$$
\left(\mathbb{E} \tilde{\mathbb{E}}\left\|\int_{0}^{\infty} \phi(t) d \tilde{W}(t)\right\|_{l^{\infty}(D)}^{p}\right)^{1 / p} \leq 6 g_{p}\left(\mathbb{E} \tau_{N}^{p / 2}\right)^{1 / p}
$$

Recall that the sequence $\left(\tau_{n}-\tau_{n-1}\right)_{n=1}^{N}$ is identically distributed. For $p=2$ we obtain

$$
\begin{aligned}
\left(\mathbb{E} \tau_{N}^{p / 2}\right)^{1 / p} & =\left(\mathbb{E} \tau_{N}\right)^{1 / 2}=\left(\mathbb{E} \sum_{n=1}^{N} \tau_{n}-\tau_{n-1}\right)^{1 / 2} \\
& =\left(\sum_{n=1}^{N} \mathbb{E}\left(\tau_{n}-\tau_{n-1}\right)\right)^{1 / 2}=\left(\sum_{n=1}^{N} \mathbb{E} \tau_{1}\right)^{1 / 2}=\sqrt{N} \sqrt{\mathbb{E} \tau_{1}}
\end{aligned}
$$

For $1 \leq p<2$ we have by Hölder's inequality,

$$
\left(\mathbb{E} \tau_{N}^{p / 2}\right)^{1 / p} \leq\left(\mathbb{E} \tau_{N}\right)^{1 / 2}=\sqrt{N} \sqrt{\mathbb{E} \tau_{1}} .
$$

Finally for $p>2$, by the triangle inequality in $L^{p / 2}(\Omega)$,

$$
\begin{aligned}
\left(\mathbb{E} \tau_{N}^{p / 2}\right)^{1 / p} & =\left(\mathbb{E}\left(\sum_{n=1}^{N} \tau_{n}-\tau_{n-1}\right)^{p / 2}\right)^{1 / p} \leq\left(\sum_{n=1}^{N}\left(\mathbb{E}\left(\tau_{n}-\tau_{n-1}\right)^{p / 2}\right)^{2 / p}\right)^{1 / 2} \\
& =\left(\sum_{n=1}^{N}\left(\mathbb{E} \tau_{1}^{p / 2}\right)^{2 / p}\right)^{1 / 2}=\sqrt{N}\left(\mathbb{E} \tau_{1}^{p / 2}\right)^{1 / p} .
\end{aligned}
$$

By Lemma 2.4 this proves that, for all $p \in[1, \infty)$ and some universal constant $K_{p}$,

$$
\left(\mathbb{E} \tilde{\mathbb{E}}\left\|\int_{0}^{\infty} \phi(t) d \tilde{W}(t)\right\|^{p}\right)^{1 / p} \leq K_{p} \sqrt{N}
$$

Since $l^{\infty}(D)$ can be identified isometrically with a finite-dimensional subspace of $c_{0}$, this completes the proof.

Corollary 2.6. Let $E$ be a Banach space. If there exists a constant $c_{p}>0$ such that for all elementary processes (2.1) holds, then E has finite cotype.

Proof. It follows from the above example that $c_{0}$ is not finitely representable in $E$. Hence the Maurey-Pisier Theorem (see [12]) implies that $E$ has finite cotype. 
Proof of Theorem 2.2. We may assume that the martingale starts at zero (see [1, Remark 1.1 ]). Let $\left(r_{n}\right)_{n=1}^{N}$ be a Rademacher sequence on the probability space $(\Omega, \mathcal{F}, \mathbb{P})$ and let $\left(d_{n}\right)_{n=1}^{N}$ be an $E$-valued martingale difference sequence with respect to the filtration $\left(\sigma\left(r_{1}, r_{2}, \ldots, r_{n}\right)\right)_{n=0}^{N}$. We may write $d_{n}=r_{n} f_{n}\left(r_{1}, \ldots, r_{n-1}\right)$ for $n=1, \ldots, N$, for some $f_{n}:\{-1,1\}^{n-1} \rightarrow E$. Let $\left(\tilde{r}_{n}\right)_{n=1}^{N}$ be a Rademacher sequence on the probability space $(\tilde{\Omega}, \tilde{\mathcal{F}}, \tilde{\mathbb{P}})$.

(1): We will show that there exists a constant $C_{p}^{-}>0$ only depending on $E$ such that

$$
\mathbb{E}\left\|\sum_{n=1}^{N} d_{n}\right\|^{p} \leq\left(C_{p}^{-}\right)^{p} \mathbb{E} \tilde{\mathbb{E}}\left\|\sum_{n=1}^{N} \tilde{r}_{n} d_{n}\right\|^{p}
$$

We use the notation of Lemmas 2.3 and 2.4. Define a process $\phi:[0, \infty) \times \Omega \rightarrow E$ by

$$
\phi(t)= \begin{cases}f_{n}\left(\Delta W_{1}, \ldots, \Delta W_{n-1}\right) & \text { for } t \in\left(\tau_{n-1}, \tau_{n}\right], n=1, \ldots, N, \\ 0 & \text { for } t=0 \text { or } t>\tau_{N} .\end{cases}
$$

The process $\phi$ is stochastically integrable and we have

$$
\begin{aligned}
& \mathbb{E}\left\|\int_{0}^{\infty} \phi(t) d W(t)\right\|^{p}=\mathbb{E}\left\|\sum_{n=1}^{N} \Delta W_{n} f_{n}\left(\Delta W_{1}, \ldots, \Delta W_{n-1}\right)\right\|^{p} \\
& \quad=\mathbb{E}\left\|\sum_{n=1}^{N} r_{n} f_{n}\left(r_{1}, \ldots, r_{n-1}\right)\right\|^{p}=\mathbb{E}\left\|\sum_{n=1}^{N} d_{n}\right\|^{p} .
\end{aligned}
$$

Also, we have

$$
\tilde{\mathbb{E}} \mathbb{E}\left\|\int_{0}^{\infty} \phi(t) d \tilde{W}(t)\right\|^{p}=\mathbb{E} \tilde{\mathbb{E}}\left\|\sum_{n=1}^{N} \Delta \tilde{W}_{n} f_{n}\left(\Delta W_{1}, \ldots, \Delta W_{n-1}\right)\right\|^{p}
$$

By Lemma 2.4, Corollary 2.6 and [10, Proposition 9.14], we have

$$
\mathbb{E} \tilde{\mathbb{E}}\left\|\sum_{n=1}^{N} \Delta \tilde{W}_{n} x_{n}\right\|^{p} \leq K_{p} \mathbb{E} \tilde{\mathbb{E}}\left\|\sum_{n=1}^{N} \tilde{r}_{n} x_{n}\right\|^{p}
$$

where $\left(x_{n}\right)_{n=1}^{N}$ is a sequence in $E$ and $K_{p}>0$ is some constant depending only on $E$ and $p$. By conditioning (cf. [8, Lemma 3.11]) this result extends to

$$
\mathbb{E} \tilde{\mathbb{E}}\left\|\sum_{n=1}^{N} \Delta \tilde{W}_{n} X_{n}\right\|^{p} \leq K_{p} \mathbb{E} \tilde{\mathbb{E}}\left\|\sum_{n=1}^{N} \tilde{r}_{n} X_{n}\right\|^{p}
$$

where $\left(X_{n}\right)_{n=1}^{N}$ is a sequence of $E$-valued random variables independent of $\left(\Delta \tilde{W}_{n}\right)_{n=1}^{N}$ and independent of $\left(\tilde{r}_{n}\right)_{n=1}^{N}$. By Lemmas 2.3 and 2.4 we may apply (2.4) to the random variables $X_{n}=f_{n}\left(\Delta W_{1}, \ldots, \Delta W_{n-1}\right)$ for $1 \leq n \leq N$ to 
obtain:

$$
\begin{aligned}
& \mathbb{E} \tilde{\mathbb{E}}\left\|\sum_{n=1}^{N} \Delta \tilde{W}_{n} f_{n}\left(\Delta W_{1}, \ldots, \Delta W_{n-1}\right)\right\|^{p} \leq K_{p}^{p} \mathbb{E} \tilde{\mathbb{E}}\left\|\sum_{n=1}^{N} \tilde{r}_{n} f_{n}\left(\Delta W_{1}, \ldots, \Delta W_{n-1}\right)\right\|^{p} \\
& \quad=K_{p}^{p} \mathbb{E} \tilde{\mathbb{E}}\left\|\sum_{n=1}^{N} \tilde{r}_{n} f_{n}\left(r_{1}, \ldots, r_{n-1}\right)\right\|^{p} \stackrel{(\mathrm{i})}{=} K_{p}^{p} \mathbb{E} \tilde{\mathbb{E}}\left\|\sum_{n=1}^{N} \tilde{r}_{n} r_{n} f_{n}\left(r_{1}, \ldots, r_{n-1}\right)\right\|^{p} \\
& \quad=K_{p}^{p} \mathbb{E} \tilde{\mathbb{E}}\left\|\sum_{n=1}^{N} \tilde{r}_{n} d_{n}\right\|^{p} .
\end{aligned}
$$

In (i), we used that $\left(r_{1}, \ldots, r_{N}, \tilde{r}_{1}, \ldots, \tilde{r}_{N}\right)$ and $\left(r_{1}, \ldots, r_{N}, r_{1} \tilde{r}_{1}, \ldots, r_{N} \tilde{r}_{N}\right)$ are identically distributed. By assumption we have

$$
\mathbb{E}\left\|\int_{0}^{\infty} \phi(t) d W(t)\right\|^{p} \leq c_{p}^{p} \mathbb{E} \tilde{\mathbb{E}}\left\|\int_{0}^{\infty} \phi(t) d \tilde{W}(t)\right\|^{p} .
$$

We may conclude that (2.3) holds with constant $c_{p} K_{p}$.

(2): We will show that there exists a constant $C_{p}^{+}>0$ only depending on $E$ such that

$$
\mathbb{E} \tilde{\mathbb{E}}\left\|\sum_{n=1}^{N} \tilde{r}_{n} d_{n}\right\|^{p} \leq\left(C_{p}^{+}\right)^{p} \mathbb{E}\left\|\sum_{n=1}^{N} d_{n}\right\|^{p} .
$$

Let $\phi$ be as before. By Lemmas 2.3, 2.4 and [10, Lemma 4.5] and the same arguments as before we have

$$
\begin{aligned}
\mathbb{E} \tilde{\mathbb{E}}\left\|\sum_{n=1}^{N} \tilde{r}_{n} d_{n}\right\|^{p} & =\mathbb{E} \tilde{\mathbb{E}}\left\|\sum_{n=1}^{N} \tilde{r}_{n} f_{n}\left(r_{1}, \ldots, r_{n-1}\right)\right\|^{p} \\
& =\mathbb{E} \tilde{\mathbb{E}}\left\|\sum_{n=1}^{N} \tilde{r}_{n} f_{n}\left(\Delta W_{1}, \ldots, \Delta W_{n-1}\right)\right\|^{p} \\
& \leq \frac{1}{\left(\mathbb{E} \tilde{\mathbb{E}}\left|\tilde{W}_{1}\right|\right)^{p}} \mathbb{E} \tilde{\mathbb{E}}\left\|\sum_{n=1}^{N} \Delta \tilde{W}_{n} f_{n}\left(\Delta W_{1}, \ldots, \Delta W_{n-1}\right)\right\|^{p} .
\end{aligned}
$$

By assumption we have

$$
\mathbb{E} \tilde{\mathbb{E}}\left\|\int_{0}^{\infty} \phi(t) d \tilde{W}(t)\right\|^{p} \leq c_{p}^{p} \mathbb{E}\left\|\int_{0}^{\infty} \phi(t) d W(t)\right\|^{p}
$$

We may conclude that (2.5) holds with constant $\frac{c_{p}}{\mathbb{E} \tilde{\mathbb{E}}\left|\tilde{W}_{1}\right|}$.

\section{ACKNOWLEDGments}

We express our thanks to Jan van Neerven and Stefan Geiss for many helpful comments and Tuomas Hytönen for his contribution to the proof of Lemma 2.4

\section{REFERENCES}

[1] D. L. Burkholder, A geometrical characterization of Banach spaces in which martingale difference sequences are unconditional, Ann. Probab. 9 (1981), no. 6, 997-1011. MR0632972 (83f:60070)

[2] D. L. Burkholder, Martingales and singular integrals in Banach spaces, Handbook of the Geometry of Banach Spaces, Vol. I, 233-269, North-Holland, Amsterdam, 2001. MR1863694 (2003b:46009) 
[3] G. Da Prato and J. Zabczyk, Stochastic Equations in Infinite Dimensions, Encyclopedia of Mathematics and its Applications, Vol. 44, Cambridge University Press, Cambridge, 1992. MR.1207136 (95g:60073)

[4] D. J. H. Garling, Brownian motion and UMD-spaces, Probability and Banach Spaces (Zaragoza, 1985), 36-49, Lecture Notes in Math. 1221, Springer-Verlag, Berlin, 1986. MR0875006 (88h:60008)

[5] D. J. H. Garling, Random martingale transform inequalities, Probability in Banach spaces VI (Sandbjerg, 1986), 101-119, Progr. Probab., Vol. 20, Birkhäuser, Boston, 1990. MR1056706 (92a:60111)

[6] S. Geiss, A counterexample concerning the relation between decoupling constants and UMDconstants, Trans. Amer. Math. Soc. 351 (1999), no. 4, 1355-1375. MR1458301 (99f:60011)

[7] P. Hitczenko, On tangent sequences of UMD-space valued random vectors, unpublished manuscript, Warsaw, 1988.

[8] O. Kallenberg, Foundations of Modern Probability, Second edition, Probability and its Applications, Springer-Verlag, New York, 2002. MR1876169 (2002m:60002)

[9] S. Kwapień and W. A. Woyczyński, Random Series and Stochastic Integrals: Single and Multiple, Probability and its Applications, Birkhäuser, Inc., Boston, 1992. MR1167198 (94k:60074)

[10] M. Ledoux and M. Talagrand, Probability in Banach Spaces. Isoperimetry and Processes, Ergebnisse der Mathematik und ihrer Grenzgebiete, Vol. 23, Springer-Verlag, Berlin, 1991. MR1102015 (93c:60001)

[11] B. Maurey, Système de Haar, Séminaire Maurey-Schwartz 1974-1975: Espaces $L^{p}$, Applications Radonifiantes et Géométrie des Espaces de Banach, Exp. Nos. I et II, 26 pp. MR 0420839 $(54: 8851)$

[12] B. Maurey and G. Pisier, Séries de variables aléatoires vectorielles indépendantes et propriétés géométriques des espaces de Banach, Studia Math. 58 (1976), no. 1, 45-90. MR0443015 (56:1388)

[13] T. R. McConnell, Decoupling and stochastic integration in UMD Banach spaces, Probab. Math. Statist. 10 (1989), 283-295. MR1057936 (91i:60010)

[14] S. Montgomery-Smith, Concrete representation of martingales, Electron. J. Probab. 3 (1998), No. 15, 15 pp. MR1658686 (99k:60116)

[15] J.M.A.M. van Neerven, M.C. Veraar and L. Weis, Stochastic integration in UMD Banach spaces, submitted.

[16] J.M.A.M. van Neerven and L. Weis, Stochastic integration of functions with values in a Banach space, Studia Math. 166 (2005), 131-170. MR2109586 (2005j:60107)

[17] V. H. de la Peña and E. Giné, Decoupling. From Dependence to Independence Randomly Stopped Processes. U-statistics and Processes. Martingales and Beyond, Probability and its Applications, Springer-Verlag, New York, 1999. MR.1666908 (99k:60044)

Delft Institute of Applied Mathematics, Technical University of Delft, P.O. Box 5031, 2600 GA Delft, The Netherlands

E-mail address: M.C.Veraar@math.tudelft.nl 